\title{
Dritte Abhandlung über Kaiser's Theorie der Muskelzuckung.
}

Von

Dr. Fr. Schenck.

Mit 1 Holzschnitt.

Ich würde mich nicht berechtigt halten, zu der Controverse zwischen Kaiser und mir nochmals das Wort zu ergreifen, wenn es sich in unserem Streit nur um Meinungsversehiedenheiten handelte ïber die Frage, ob die Massentbeilchen des unbelasteten Muskels durch die contractilen Kräfte geschleudert werden oder nicht. Aber die Sache liegt anders. Ich behaupte, den unanfechtbaren Beweis erbracht zu haben, dass die Begründung, die $\mathrm{Ka}$ a i e r seiner Hypothese giebt, physikalis $\mathrm{ch}$ falsch ist. Kaiser versucht neuerdings ${ }^{1}$ ) nochmals, zu zeigen, dass ich diesen Beweis nicht erbracht habe und er kündigt sogar eine grössere zusammenfassende Darstellung seiner Lehre an. Da dürfte es mir wohl gestattet sein, auch noch einmal mitzusprechen.

Ich glaube aber auch antworten zu müssen, um meinem Gegner gerecht zu werden, da derselbe mir den Vorwurf macht, ich hätte die Ergebnisse seiner Muskelversuche, die er für seine Theorie beweisend ansieht, unberüeksichtigt gelassen.

Trotzdem wïrde ich das Wort nicht ergreifen, wenn ich nicht auch aber $\mathrm{n}$ e $\mathrm{u}$ e Versuche am unbelasteten Muskel berichten könnte, die zu unserer Streitfrage in Beziehung stehen.

I. Die physikalischen Grundlagen der Theorie Kaisers.

1. Wenn man eine senkrecht aufgehängte Spiralfeder durch Zug am unteren Ende dehnt, dann plötzlich entlastet, so geht das

1) Zeitschr, f. Biolog. Bd. 35. S. 94. 
untere Ende zunächst tuber seine Ruhelage in Folge Schleuderung der Massentheilchen noch hinaus und stellt sich erst nach einigen Schwingungen auf- und abwärts in seine Ruhelage wieder ein. In Figur 1 sei der zeitliche Verlauf der Verkürzung einer Spiralfeder durch die aufeinander folgenden Längen $A_{1} B_{1}$, $A_{2} B_{2}, \ldots$ dargestellt. $A_{2} B_{2}$ bedeute die Ruhelänge, die Höhe $B_{2}$ entspricht also dem „zweiten Fusspunkt" Kaiser's. $A_{1} B_{1}$ sei die Länge, bis zu der die Feder vor der Entlastung gedehnt war, $A_{4} B_{4}$ ist die Feder im Zustand grösster Verkürzung bei der ersten Auf-

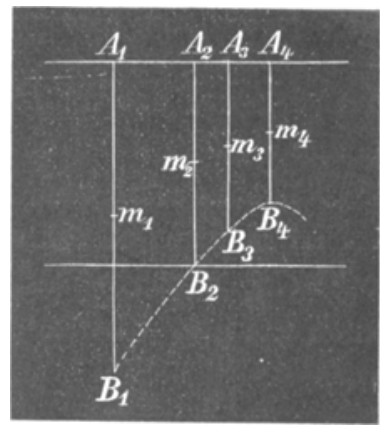
wärtsschwingung. Ein Massentheilchen $M$ der Feder in der Mitte zwischen beiden Enden nimmt dann nacheinander die Lagen $M_{1}$, $M_{2}, M_{3}, \ldots$ ein.

Gesetzt, ich halte das untere Federende $B$, während es sich ans der Höhe $B_{2}$ zur Höhe $B_{4}$ bebt, etwa in $B_{3}$ plötzlich fest, so dass es sich nicht mehr weiter anfwärts, wohl aber wieder abwärts bewegen kann, wie ändert sich dann die Bewegung von $B$ und $M$ ?

Es wird nicht etwa auch $M$ an seiner weiteren Aufwärtsbewegung gehemmt, denn damit $M$ festgehalten würde, müsste zwischen $B$ und $M$ eine undehnbare Verbindung sein. Das ist nicht der Fall: die Verbindung ist die untere Hälfte der dehnbaren Feder. Ueberdies ist im Moment der Hemmung die Feder in ihrer Längsrichtung zusammengedrückt. Durch Festhalten von $B$ in $B_{3}$ kann man also auf $M$ nicht einmal einen durch Dehnung der Feder allmählich zunehmenden $\mathrm{Zug}$ abwärts ausuben, weil dazu der Abstand zwischen $\boldsymbol{B}$ und $\boldsymbol{M}$ mindestens gleich der Ruhelänge der unteren Federhälfte sein müsste.

Also wird die Bewegung von $M$ nicht von unten her gehemmt.

Dasselbe, was für $M$ gesagt ist, gilt für alle oberhalb und unterhalb $M$ gelegene Massentheilchen. Für jedes Theilchen gilt auch der Satz, dass es nach der Hemmung sich weiter aufwärts bewegen kann, so lange, bis der Theil der Feder unter ihm die Ruhelänge erreicht bat; aber selbst danach bewegt es sich noch aufwärts weil es nun durch seine lebendige aufwärts treibende Kraft das nntere Federstück dehnt. Dadurch wird allerdings ein allmählicb 
zanehmender $\mathrm{Zug}$ abwärts hergestellt, der im Verein mit dem von oben wirkenden Druck bewirkt, dass das Theilchen weniger hoch steigt, als bei ungehemmter Bewegung.

Nach der Hemmung gehen alle Massentheilchen zunächst also weiter aufwärts, ein Theil von ihnen übt dabei einen $\mathrm{Zng}$ aufwärts auf das untere Federende aus. Dieses Ende bleibt daher einige Zeit in der Höhe $B_{3}$ stehen, um danach erst zu sinken.

$\mathrm{Ka}$ is er ist anderer Ansicht. Er glaubt, dass die Massentheilchen und das Federende nach der Hemmung sofort umkehren, weil ein „abwärts wirkender Stoss", der sich zu der sehon ohnehin nach abwärts wirkenden Druckelasticität addirt, die Umkehr bewirkt ${ }^{1}$ ).

Die Druckelasticität selbst ist es also nicht, die nach Kais er's Ansicht die Massentheilchen abwärts stösst; sie kann das ja auch nicht, weil sie kleiner ist als die entgegenwirkende lebendige Kraft der Massentheilchen.

Ich frage Kaiser: Woher kommt der abwärts gerichtete Stoss? Ich bitte ihn aber dringend, diese schon friher ${ }^{2}$ ) an ihn gerichtete Frage nicht nochmals mit Stillschweigen zu übergehen, weil sie für unsere Controverse von entscheidender Bedeutung ist. Wenn er den Nachweis bringt, dass der Stoss erfolgen muss, will ich mich für geschlagen erklären.

2. Realisirt man den Federversuch derart, dass man an das untere Federende einen Schreibhebel bängt, der die Hemmung bewirkt durch Anstossen gegen einen über ihm eingestellten Anschlag, so wird der Versuch dadurch complicirt, dass der Hebel Eigenbewegungen ausführen kaun. Stösst der Hebel gegen den Anschlag an, so prallt er - allein für sich betrachtet - ab und sinkt sofort wieder abwärts. Nur wenn die Kraft, mit der der Hebel zurïckprallt, kleiner ist als der Zug der Massentheilchen aufwärts, bleibt der Hebel einige Zeit am Anschlag liegen. Zieht die Hebelmasse stärker abwärts, als die Massentheilchen anfwärts, so muss der Hebel sofort rom Anschlag absinken. Das sofortige Absinken des Hebels rom Anschlag beweist aber noch nicht einmal, dass nun auch eine sofortige Umkehr aller Massentheilchen eingetreten ist, weil der Fall möglich ist, dass trotz des Absinkens

1) Zeitschr. f. Biolog. Bd. 333. S. 353.

2) Dies Archiv Bd. 65. S. 319. 
des Hebels die oberen Massentheilchen der Feder sich nocb weiter aufwärts bewegen, indem das unter ihnen befindliche Federstïck gedehnt wird.

Wenn also in einem Federversuche der Hebel sofort vom Anschlage absinkt, so muss die Möglichkeit ausgeschlossen sein, dass das Absinken durch Eigenbewegungen des Hebels erfolgt, wenn der Versuch für Kaiser's Ansicht beweisend sein soll. Da nun Kai s e r für keinen einzigen seiner Federversuche, auf die er sich beruft, auch nicht für seine in der jüngsten Abhandlung erwähnten, bisher diesen Nachweis erbracht hat, so sind alle diese Versuche werthlos zur Entscheidung unserer Streitfrage. Kaiser beruft sich von neuem immer wieder auf Federversuche, von denen ich schon längst ${ }^{1}$ ) gezeigt habe, dass sie nichts für ihn beweisen.

Von den Bedingungen aber, die Kaiser jetzt zum Gelingen der Federversuche in seinem Sinne günstig erklärt, lässt sich unschwer zeigen, dass sie nur desshalb für $\mathrm{Ka}$ is er giunstig sind, weil sie ein Ueberwiegen des Zuges der Hebelmasse über den Zug der Massentheilchen der Feder begiunstigen.

3. Gegen mein e Federversuche ${ }^{2}$ ), deren Resultat für die Richtigkeit meiner physikalischen Deductionen spricht, wendet $\mathrm{K}$ a is er jetzt Folgendes ein: Meine Spiralfeder soll zu lang, der Abstand ihrer Windungen za gross gewesen sein. Solch lange Federn weichen nach $\mathrm{K}$ a is e $\mathrm{r}$,gewissermaassen der Wirkung des in senkrechter Richtung erfolgenden Stosses ans, indem sie Schwingungen um die auf den Windungsflächen senkrecht stehende Längsachse ausführen. Die Federn krümmen sich also und halten dadurch den Hebel eine Zeit lang an der Hemmung fest."

Dazu bemerke ich: Der von Kaiser angenommene Stoss, der stärker sein soll, als die lebendige Kraft der Massentheilchen, miisste unter allen Umständen die Massentheilchen zur sofortigen Unkehr nach unten zwingen, einerlei ob die Bewegungsrichtung der Massentheilchen vorher genau senkrecht oder mehr weniger schräg aufwärts erfolgt. Das ergiebt die allereinfachste Construction des Parallelogramms der Kräfte. Ein seitliches Ausweichen der Massentheilchen vor dem Stoss ist mechanisch unmöglich.

Sollte also eine Seitwärtsbewegung in meinen Federversuchen

1) Dies Archiv Bd. 63. S. 361 u. Bd. 65. S. 323.

2) Dies Archiv Bd. 63. S. 359. 
vorgekommen sein, so liegt darin kein Versuchsfehler. Meine Versuche sind nach wie vor beweisend für meine Auffassung, und da Ka is er sie jetzt bestätigt hat, so hat er selbst die Richtigkeit meiner physikalischen Deductionen experimentell bewiesen.

4. Unsere Federversuche, von denen ich bisher gesprochen habe, sind Ka iser's Verlangen entsprechend angestellt mit Hebeln, die so leicht waren, dass sie die Federn nur unmerklich belasteten. Nun hat $\mathrm{Kaiser}$ schon früher Federversuche angestellt mit Hebeln, die nach isotonischem Principe stärker belastet waren und daher die Feder um ein Beträehtliches dehnten. Auch in den Versuchen mit belasteten Federn hat $\mathrm{K}$ a iser das Liegenbleiben des Hebels am Anschlag beobachtet, allerdings nur dann, wenn der Anschlag so eingestellt war, dass im Moment des Anschlages die Feder immerhin noch länger war, als in ganz ungedehntem Zustande. $\mathrm{K}$ a iser glaubt jedoch, dass das Ergebniss dieser Versuche nicht die Richtigkeit meiner physikalischen Deductionen beweist, weil das Liegenbleiben des Hebels nicht durch die Trägheitsbewegung der Massentheilcben, sondern durch die in der Feder noch wirksamen elastischen Verkürzungkräfte bewirkt sei.

Ich will durchaus nicht leugnen, dass die von der Last wach gerufenen elastischen Kräfte überhaupt verkürzend wirken können, wenn die Last in die Höhe geworfen wird, aber ich bestreite ganz entschieden, dass in unserem speciellen Falle jene Kräfte die Last auf der Anschlaghöhe einige Zeit halten. Die elastischen Kräfte sind in diesem Falle kleiner als die Last. Im Moment des Anschlages hört die Aufwärtsbewegung der Last auf; von da ab wirkt also die Last wieder mit dem vollen Betrage ihrer Schwere auf die Feder und müsste sie s of o $\mathrm{t}$ bis zur Ruhelänge dehnen. Wenn der Hebel nun doch noch einige Zeit am Anschlag liegen bleibt, so kann das nur dadureh geschehen, dass die aufwärts geschleuderten Massentheilchen den Zug nach oben zu Stande bringen. Ein Unterschied in der Wirkung des Anschlages bei unbelasteter und belasteter Feder besteht also nicht, daher darf auch für die belastete Feder der zweite Fusspunkt in der Ruhelage des unteren Federendes angenommen werden. Wenn $\mathrm{K}$ a i s e $\mathrm{r}^{1}$ ) hiergegen geltend macht, dass nach seiner Definition der zweite Fusspunkt in allen Fällen durch die Lage des unteren

1) Zeitschr. f. Biolog. Bd. 33. S. 159. 
Endes der unbelasteten Feder angegeben wird, so ibersieht er, dass diese Definition in Widerspruch steht mit derjenigen Definition, die sich als logische Consequenz aus seiner Methode der Bestimmung des zweiten Fusspunkts ergiebt.

II. Kritik der Muskelversuche, die Kaiser für seine Theorie beweisend bält.

Kai s er leugnet, dass die Ergebnisse der Muskelversuche, auf die er seine Theorie von der Schleuderung der Massentheilchen der Muskeln stützt, bedingt sein können durch Wurf des Zeichenhebels. Dem Verlangen Kaiser's entsprechend, will ich hier zeigen, dass das wohl der Fall ist.

Auf vier Thatsachen stitzt $\mathrm{Ka}$ is e $\mathrm{r}$ seine Ansicht ${ }^{1}$ ). In den folgenden Abscbnitten 5 bis 8 will ich diese Thatsachen nacheinander discutiren.

5. Ka is er sagt: "Wenn die Abwärtsbewegung des Hebels nach dem Anschlag im zweiten Fusspunkt bedingt wäre durch Schleuderung des Hebels gegen die Hemmung und Abprall von derselben, so wäre die Linie $a b$, Fig. $8^{2}$ ), (d. i. die Linie, die der Hebel nach dem Anschlag zeichnet) die Curve eines senkrecht nach abwärts gerichteten Wurfes. Je höher der Anschlag über dem zweiten Fusspunkt erfolgt, desto geringer ist die Geschwindigkeit, mit der der Hebel gegen den Anschlag stösst", desto flacher müsste die Wurfeurve also werden.

Hierzu bemerke ich: Was Kaiser hier im Sinne meiner Ansicht postulirt, ist auch thatsächlich der Fall im allerersten Anfang (etwa im ersten Millimeter) des Abfalls nach dem Anschlag. Kaiser beriicksichtigt zur Beurtheilung der von ihm aufgeworfenen Frage nur die späteren Theile der Curve; das ist unzulässig, weil hier auf den Verlauf des Abfalls der Curve auch von Einfluss ist der Verkürzungszustand des Muskels in dem Moment, wo der Muskel den herabsinkenden Hebel wieder auffängt. Der Muskel verlängert sich vom zweiten Fusspunkt ab allmählich wieder, er wird den Abstieg des Hebels verzögern, wenn er den Hebel wieder auffängt. Der Hebel trifit aber um so früher auf den

1) Zeitschr. f. Biolog. Bd. 33. S. 356.

2) Zeitschr, f. Biolog. Bd. 33. Tafel V. 
Muskel, sein Abstieg wird also um so eher verzögert, je früher der Anschlag erfolgt ist.

6. Kaiser sagt, wenn Hebelschleuderung in seinen Versuchen im Spiele gewesen wäre, müsste der absteigende Schenkel der ohne Anschlag gezeichneten Curve die dem freien Fall entsprechende Geschwindigkeit erreichen. Eine zur Controle gezeichnete Falleurve des Hebels verläuft aber, wie er behauptet, steiler, als der Abstieg der Muskelcurven.

Darauf entgegne ich: Kaiser's Figuren lehren etwas Anderes, als er im Text der Abhandlung sagt. Wenn man unter Bericksichtigung der Verschiedenheiten des Zeitmaassstabes - die Trommelgeschwindigkeit ist bei der Falleurve kleiner als bei den meisten Muskelcurven - die Fallcurve, mit s ämmtlichen Muskelcurven $\mathrm{Ka}$ iser's, nicht nur mit den auf Tafel V, Zeitschr. f. Biol. Bd. 33 befindlichen, vergleicht; so sieht man, dass die Muskelcurven nicht immer langsamer, oft sogar schneller abfallen, als die Hebelfalleurve. Auf Tafel VI und VII 1. e. Bd. 33 befinden sich viele Muskelcurven, deren Abstieg steiler ist, als die Hebelfallcurve von Tafel V. Dass der Abstieg der Muskelcurve manchmal steiler, manchmal flacher ist, als der der Falleurve, könnte durch Verschiedenheiten der Reibung der Schreibspitze an der Schreibfläche in den einzelnen Versuchen bedingt sein.

Genaueres darüber lässt sich allerdings nicht sagen, weil in den Muskelversuchen die mechanischen Bedingungen des Falls noch dadurch complicirt sind, dass mit dem Hebel zugleich das Verbindungsstïck und sogar der Maskel, falls er gewackelt haben sollte, fallen.

7. Ka i s e r sagt, wenn in seinen Versuchen Hebelschleuderung stattgefunden hätte, hätte der Hebel vom Anschlag sofort absinken müssen, wenn der Anschlag im steilsten Stück des Anstiegs der Zuckungseurve erfolgt, weil an der steilsten Stelle die Sehleuderung beginnt. Kaiser hat aber den Anseblag höher einstellen müssen, um sofortiges Absinken za bekommen.

Darauf entgegne ich: Wenn auch der Hebel sich vom Momente der grössten Steilheit ab frei bewegt, so kann sich von da $a b$ auch der Muskel noch einige Zeit weiter verkürzen, ohne den Hebel noch zu beschleunigen. Wenn also der Anschlag über der Stelle grösster Steilheit erfolgt, so ist es doch möglich, dass der Hebel 
nicht sofort absinkt, weil er gleich danach wieder von den aufwärts ziehenden contractilen Kräften erfasst wird.

Was Kaiser hier betreffs der Hebelschleuderung sagt, würde übrigens, wenn es richtig wäre, auch für die von ihm angenommene Schleuderung der Massentheilchen des Muskels zutreffen.

8. Ka iser schreibt: „Wenn ich den Hebel an der Achse so belaste $(15 \mathrm{gr})$, dass der Gipfel der Zuckungscurve mit dem zweiten Fusspunkt zusammenfällt und entlaste auf dem Gipfel, so tritt keine sogenannte Entlastungszuckung auf, sondern der Hebel sinkt in stetigem Zuge zur Abscisse ab. Wenn auch diese Erscheinung auf Schleuderung des Hebels zurückgeführt werden soll, so müsste der absteigende Schenkel nach der Entlastung einer Fallcurve entsprechen, er ist aber flacher als die Fallcurve".

Diese Beobachtung Kaiser's erklärt sich so: Da der Hebel im Beginn des Versuches nach isotonischem Principe belastet ist, so wird er weniger geschleudert, als wenn er ganz unbelastet verwendet worden wäre und nur etwa bis zur Höhe geworfen, die der Hubhöhe des unbelasteten Muskels entspricht. Durch diesen Wurf entlastet sich der Muskel während der Contraction selbst und verkürzt sich auch etwa bis zn dem Grade, der der Hubhöhe des unbelasteten Muskels entspricht. Das, was für meine Anschauungen Hubböhe des unbelasteten Muskels ist, ist ja im Sinne der Theorie Kaiser's die Höhe des zweiten Fusspunctes. Bleibt nun auf dem Gipfel die Last am Hebel hängen, so dehnt sie beim Abfall den Muskel wieder; wird auf dem Gipfel die Last abgenommen, so beschreibt der Schreibhebel nun einfach die Curve, die die Wiederverlängerung des unbelastet zuckenden Muskels angiebt. Dass diese Wiederverlängerung langsamer erfolgt, als der Fall, ist nach unseren bisherigen Erfahrungen eigentlich selbstverständlich, und desshalb bieten die Entlastungsversuche Kaiser's gar nichts Interessantes.

III. Ueber Kaiser's und meine Versuche mit Unterstützung des Hebels im zweiten Fusspunct.

9. Um zu zeigen, dass in Kai ser's Versuchen Hebelscbleuderungen im Spiele gewesen sind, habe ich seine Versuche nachgemacht ${ }^{1}$ ), dabei aber vor der Zuckung den Hebel in der Höhe

1) Dies Archiv Bd. 65. S. 324. 
des zweiten Fusspunctes unterstützt. Er wurde nicht mebr durch die Zuckung gehoben. Daraus folgt, dass in den Versuchen ohne Unterstützung der Hebel geschleudert wurde; nach $\mathrm{Kaiser}$ 's Theorie bätte auch der unterstitzte Hebel über den zweiten Fusspunct gehoben werden müssen.

$\mathrm{Ka}$ i s e $\mathrm{r}$ wendet ein, dass meine Versuchsbedingungen für Hebelschleuderung günstiger gewesen sind, als seine. Ich will demgegenüber zeigen, dass meine Versuchsbedingungen nicht günstiger, sondern im Gegentheil eher ungïnstiger für die Hebelschleuderung sind, als die Versuchsbedingungen $\mathrm{K}$ a is e $\mathrm{r}$ 's.

$\mathrm{K}$ a i s e r verbindet den Muskel mit dem Hebel durch einen Draht, ich durch ein Haar, um eine möglichst leichte biegsame Verbindung za haben. $\mathrm{K}$ aise $\mathrm{r}$ behauptet, durch die Dehnbarkeit des Haares werde die Hebelschleuderung begiunstigt. Nun, abgesehen davon, dass die Dehnbarkeit des Haares bei der geringen Hebelmasse - der Hebel belastete den Muskel mit 0,2 gr -- nicht in Betracht kommt, würde die Dehnbarkeit des Haares nicht günstig, sondern ungünstig für Hebelschleuderung sein. Die Spannung, die zur Ueberwindung der Trägheit des Hebels entwickelt werden muss, entsteht schneller bei undehnbarem, als bei dehnbarem Verbindungstick, weil zum Anspannen des letzteren Zeit verloren geht. Der Hebel wird desshalb in ersterem Falle schneller bewegt, mithin mehr geschleudert, als in letzterem.

Mein Schreibhebel ist ungüinstiger für Hebelschleuderung, als der von Kaiser zu seinen alten Versuchen verwendete, weil er leichter ist $(0,2 \mathrm{gr}$ statt $1,25 \mathrm{gr}$ als Last am Muskel) und weniger stark vergrössert (4fach statt 6,66 fach).

Dass ich meine Muskeln erwärmt habe, nm ein erbebliches Ueberschreiten des zweiten Fusspunctes zu bekommen, darf mir $\mathrm{K}$ a i s e $\mathbf{r}$ nicht als Fehler vorhalten, weil er seine Versuchsresultate von erwärmten Muskeln auch auf die inneren Schleuderungen beziebt. Unregelmässigkeiten der Hubhöhen des erwärmten Maskels habe ich bei meinen vielen Versuchen über den Einfluss der Temperatur auf den Muskel nie beobachtet; die Bemerkungen, die Kaiser darüber macht, treffen für meine Versuche nicht zu. Uebrigens wïrde es sehr merkwïrdig sein, wenn immer gerade bei den Unterstiutzungszuckungen die Hubhöhe klein, bei den anderen zum Vergleich gezeichneten gross gewesen wäre.

$\mathrm{Da}$ in meinen Unterstïtzungsversuchen Hebelschlenderung 
mehr vermieden ist, als in den früheren Versuchen $\mathrm{K}$ a i s e r's, und da Kaiser selbst anerkennt, dass in meinen Versuchen der Hebel geschlendert wurde, so giebt er mithin indirect auch für seine Versuche die Hebelschleuderung zu.

10. In der Beschreibung meiner Unterstützungsversuche babe ich darauf aufmerksam gemacht, dass der im zweiten Fusspunct unterstützte Hebel noch durch die Zuckung geboben werden kann, wenn das untere Muskelende sich in Folge Eigenbewegingen des ganzen Muskels, die ich kurz "Wackeln" nannte, hïher erhebt als der physiologischen Hublöbe entspricht. Auf das Wackeln führe ich auch die Thatsache zurück, dass Ka is er ${ }^{1}$ ) die Erhebung des unterstïtzten Hebels über den zweiten Fusspunct beobachtet hat, als er meine Unterstützungsversuche nachmacbte. $\mathrm{K}$ a i s e r behauptet, das Wackeln durch sorgfältige Präparation der Muskelinsertion am Oberschenkel vermieden zu haben. Damit ist aber nichts gewonnen, weil das Wackeln nicht durch die Art, wie der Muskel am Knochen befestigt ist, bedingt wird, sondern durch den unregelmässigen Bau und die ungleichmässige Contraction in verschiedenen Theilen des Muskels. Die Möglichkeit, dass der frei hängende Muskel wackelt, ist überhaupt nicht von vorne herein sicher auszuschliessen.

Zur Beurtheilung der Unterstïtzungsversuche, in denen der unterstïtzte Hebel über den zweiten Fusspunkt erhoben wird, muss auch noch folgende Möglichkeit bedacht werden, auf die ich schon frïher ${ }^{2}$ ) aufmerksam gemacht habe: Ein sofortiges Absinken des Hebels vom Anschlag kann selbst dann vorkommen, wenn im Moment des Anschlags noch contractile Kraft im Muskel wirkt. Das geschieht, wenn die Kraft des zurückprallenden Hebels grösser ist als die entgegenwirkende contractile Kraft. Dann wirkt also noch contractile Kraft im Muskel, selbst nachdem der durch Ka iser's Methode gefundene zweite Fusspunkt überschritten ist.

Nach Kaiser's Theorie erhalten die Massentheilchen des Muskels beim Anschlag einen Stoss nach abwärts, der auf den Hebel uibertragen wird. Nun benutzt er jetzt ein Verbindungstück zwischen Muskel und Hebel, das den Stoss gar nicht übertragen kann, nämlich einen Seidenfaden. Trotzdem fällt der Hebel nicht vom An-

1) Zeitschr. f. Biolog. Bd. 35. S. 98.

2) Dies Archiv Bd. 61. S. 77. 
schlag abwärts, sondern er wird abwärts gestossen. Der Stoss, den der Hebel erbalten hat, kann also nur durch den Anprall am Anschlag bewirkt sein. Dadureh hat $\mathrm{Ka}$ iser selbst den schönsten Beweis erbracht, dass in seinen neuen Versuchen Hebelschleuderung resp. das für unsere Betrachtung der Hebelschleuderung: gleichbedeutende Wackeln des Muskels im Spiele war.

IV. Ueber einige Versuchsresultate Kaiser's, die gegen seine eigene Theorie sprechen.

11. Die Schwingungen der Massentheilehen im Sinne der Theorie Kaiser's erfolgen in seinen Muskelversuchen in erheblicher Grösse nur bei der ersten Aufwärtsbewegung nach der Contraction. Schon bei der ersten Abwärtsschwingung geht der Hebel aperiodisch in seine Gleichgewichtslage, d. i. die Abscissenachse uiber. Denn die kleinen Schwingungen am Ende der Zuckungscurve sind zu vernachlässigen gegenüber der colossalen ersten Aufwärtsschwingung. Die Massentheilchen des Muskels werden also nach $\mathrm{Ka}$ ise $\mathrm{r}$ im aufsteigenden Theile der Zuckungscurve enorm geschleudert - dann ist auf einmal ihre Bewegung geradezu vollkommen gedämpft. Wie reimt sich das zusammen?

Man könnte fragen, ob nicht auch bei Hebelschleuderung die Schwingungen am Ende der Zuckungscurve grösser sein müssten, als es in $\mathrm{K}$ a i ser's Versuchen der Fall war. Das braucht nicht der Fall zu sein, weil der Hebel sehr leicht ist und daher, wenn er abwärts sinkt, die Muskelmasse nicht erheblich dehnt.

In der Curve Figur 3, Tafel II, Zeitschr. f. Biol. Bd. 33, liegt der Gipfel $28 \mathrm{~mm}$ über, die Abscissenachse $17 \mathrm{~mm}$ unter dem zweiten Fusspunct. Im Sinne der Theorie $\mathrm{K}$ a i s e r's heisst das: Die durch Zusammendrüicken des Muskels geweekte elastische Kraft ist hier grösser, als die Verkürzungskraft, aus der sie entsteht. Dürfte ich $\mathrm{K}$ a i s er bitten, auf diesen Fall die Betrachtung ${ }^{1}$ ) anzuwenden, die er - unberechtigter Weise - gegen meine Federversuche vorbringt. Er ist dann gezwungen, seine eigene Theorie als Absurdität zu bezeichnen, wenn er den von ihm selbst an der citirten Stelle gewählten Ausdruck auf sie anwendet.

1) Zeitschr. f. Biolog. Bd. 33. S. 355. Zeile $13 \mathrm{ff}$. von oben. 
V. Neue Versuche über den Einfluss der Temperatur auf die $H u b h \ddot{b}$ e des unbelasteten Muskels.

12. In der Lehre $\mathrm{K}$ a is e $\mathbf{r}$ 's könnte, auch wenn sie falsch begriindet ist, doch eins richtig sein. Es könnte seine Methode der Bestimmung des zweiten Fusspuncts dienen zur Bestimmung der Hubhöhe des unbelasteten (d. b. nur mit dem Schreibhebel belasteten) Muskels. Nun giebt $\mathrm{K}$ a i s e an, dass der zweite Fusspunct bei verschiedenen Temperaturen gleich hoch liegt. Mithin wäre die Hubhöbe des unbelasteten zuckenden Muskels bei allen Temperaturen gleich.

Um diese Folgerung nachzuprüfen, habe ich Herrn Dr. Franz Mü $1 \mathrm{l}$ e r veranlasst, mit einem Hebel, der den Muskel fast gar nicht belastete und bei welchem Schleuderung hinreichend ausgeschlossen war, die Hubhöhe des zuckenden Gastrocnemius von Rana temporaria bei directer und maximaler Reizung und bei verschiedenen Temperaturen zu bestimmen.

Die Hebelschleuderung wurde vermieden dadurch, dass der Hebel sich nicht in der üblichen Art in einer Achse drehte, sondern an dem Stativ befestigt war durch ein federndes Metallplättchen, das er bei seiner Auf- und Abwärtsbewegung verbog. Die Spannung des Federchens, die der contractilen Kraft als Gegenkraft dient, ist der Hebelschleuderung weniger günstig, als die Belastung mit einem Gewicht. Die Anfangsspannung des Muskels wurde zu 0,33 gr gewählt, die Zunahme der Spannung, die wäLrend der Contraction durch weitere Verbiegung der Feder erfolgte, betrug allerhöchstens $0,23 \mathrm{gr}$, so dass die Spannung des Muskels auch auf der Höle der Contraction nicht $0,56 \mathrm{gr}$ überstieg. Der Hebel zeichnete mit etwa zweifacher Vergrösserung.

Ob Hebelschleuderung aufgetreten war, wurde immer nach der vorhin erwähnten Unterstiitzungsmethode geprüft. Es ergab sich, dass $\mathrm{n}$ i e Hebelschleuderung erfolgt war. Bemerkenswerth ist das, wẹil als Verbindungsstück zwischen Muskel und Hebel wieder ein Haar verwendet wurde. K a i s e r's Befürchtung, das Haar bewirke Hebelschleuderung, ist also grundlos.

Die Versuche ergaben, dass die Temperatur auf die Hubhöhe auch des unbelasteten Muskels Einfluss hat und zwar in demselben Sinne, wie es $\mathrm{Gad}$ und $\mathrm{H}$ e y $\mathrm{man}^{1}$ ) für den stärker be-

1) Du Bois-Reymond's Archiv. Suppl. 1890. S. 59. 
504 Fr. Schenck: Dritte Abhandl. über Kaiser's Theorie d. Muskelzuckung lasteten Muskel angeben. Es fand sich ein relatives Minimum der Hubhöhe bei etwa $19^{\circ}$ C., zwei Maxima bei $3-4^{0}$ und bei 30 bis 350. Die Hubhöhe des warmen Muskels war meist etwas grösser, als die des kalten ${ }^{1}$ ).

Fraglich bleibt, ob das mehrfach erwähnte Wackeln des unbelasteten Muskels auf das Resultat von Einfluss gewesen sein könnte. Das Wackeln erfolgt um so mehr, je schneller der Muskel sich contrahirt. Dass bei $30-35^{0}$ die Hubhöhe grösser ist als bei $19^{0}$ könnte durch Wackeln mitbedingt sein. Das Wackeln kann aber nicht die Ursache der grösseren Hubhöhe bei $3-4^{0}$ sein. Mithin ist die Folgerung aus $\mathrm{K}$ a is er's Versuchen, dass die Temperatur keinen Einfluss auf die Hubhöhe des unbelasteten Muskels hat, nicht richtig.

13. K a i s e $\mathbf{r}$ kündigt an, dass er demnächst eine Methode der Bestimmung des zweiten Fusspunets mittbeilen wird, welche Hebelschleuderung ausschliesst. Nun, es genïgt nicht, nur die Hebelschleuderung auszuschliessen; es muss auch das Wackeln des Muskels sicher ausgeschlossen werden.

Sollte der Nachweis der inneren Sehleuderung auf ganz anderem Wege, als bisher, gelingen, so bleiben doch alle Einwände, die ich gegen die bisher geübte Methode vorgebracht habe, bestehen. Ich erkläre nämlich die bisherige $B$ e grii $n$ d u $\mathrm{ng}$ der Theorie für falsch, nicht die Theorie selbst. Ueber letztere habe ich nur gesagt, dass sie mir unwahrscheinlich ist, weil manches dafür spricht; dass die Bewegung der Massentheilchen vollkommen gedämpft ist. Nur diesen letzten Satz würde ich eventuell preisgeben müssen, sonst nichts.

1) Ausführlichere Mittheilungen in der Dissertation des Herrn Dr. Müller. Würzburg 1897.

2) Zeitschr. f. Biolog. S. 96 Zeile 16 ff. von oben. 